\title{
Entre émancipation symbolique et reproduction sociale
}

Ethnographie d'une formation de « développement personnel »

Between symbolical emancipation and social reproduction. Ethnography of a "personal development" course

\section{Hélène Stevens}

\section{OpenEdition}

\section{Journals}

Édition électronique

URL : http://journals.openedition.org/travailemploi/5945

DOI : 10.4000/travailemploi.5945

ISSN : 1775-416X

Éditeur

DARES - Ministère du Travail

Édition imprimée

Date de publication : 15 mars 2013

Pagination : $39-51$

ISSN : 0224-4365

\section{Référence électronique}

Hélène Stevens, "Entre émancipation symbolique et reproduction sociale », Travail et Emploi [En ligne] 133 | janvier-mars 2013, mis en ligne le 01 janvier 2015, consulté le 01 mai 2019. URL : http:// journals.openedition.org/travailemploi/5945 ; DOI : 10.4000/travailemploi.5945 


\title{
Entre émancipation symbolique et reproduction sociale. Ethnographie d'une formation de udéveloppement personnel »
}

\author{
Hélène Stevens $\left.{ }^{*}\right)$
}

\begin{abstract}
Derrière un objectif de formation se trouve une réalité plurielle. Que ce soit les responsables qui la conçoivent, les consultants qui l'animent ou les salariés qui la suivent, tous ces acteurs agissent sur la formation et y investissent des dispositions, des convictions, des interrogations et des situations professionnelles et personnelles. L'article analyse ainsi en contexte et de manière longitudinale les formes d'appropriation d'une formation de "développement personnel» dans une grande entreprise informatique, entre le moment de sa création et celui de ses effets quelques mois ou années après la participation des salariés. Il interroge dès lors l'écart éventuel entre les effets attendus et les effets observés, au niveau individuel comme collectif et social.
\end{abstract}

Fin des années 1990 chez un grand constructeur informatique. Après plus d'une décennie de restructurations industrielles et de plans sociaux, alors que l'accent est désormais mis sur les services au client, la direction d'entreprise appelle chacun de ses salariés à se transformer en "acteur autonome et responsable de son devenir personnel» ${ }^{(1)}$. L'entreprise Siris, anciennement soutenue par des politiques étatiques, fait savoir qu'elle ne peut plus garantir de perspectives de carrières promotionnelles ascendantes ni assurer la pérennité des emplois. Elle promeut un nouveau modèle d'emploi fondé sur la mobilité, la responsabilisation et l'autonomie des salariés. Venant soutenir ce discours, une formation appelée Entreprise de Soi est mise en place, proposant aux salariés des séminaires dits de «développement personnel». Les volontaires doivent en choisir trois parmi une offre de dix ${ }^{(2)}$ et signer un contrat établissant la confidentialité des propos échangés en cours de formation et les engageant à faire un bilan personnel de leur stage. Les séminaires, de deux jours chacun, sont animés par des consultants extérieurs à l'entreprise, dont les pratiques s'inspirent de différentes techniques

(*) Université de Poitiers - GRESCO EA3815; helene. stevens@univ-poitiers.fr

(1) Extrait de l'éditorial par le DRH du journal interne de Siris, novembre 1996.

(2) Les séminaires abordent différents thèmes, aux intitulés parlants : "Moi et mes compétences», "Moi et ma façon d'agir », "Moi et mon scénario de succès», "Moi et mon image personnelle», "Ma relation au changement", "Moi et ma dynamique de temps", "Moi et les autres", "Moi et le récit de ma vie professionnelle», "Moi, mes valeurs et le changement», "Moi et la mise en scène de moi», "Moi et la gestion du stress », "Moi mes choix et l'orientation». psychologiques telles que l'analyse transactionnelle, la programmation neuro-linguistique (PNL), l'activation et le développement vocationnel de la personne, etc. Ils peuvent être prolongés par des entretiens avec un "conseiller en stratégie de vie», destinés à approfondir la réflexion amorcée lors des séminaires et à accompagner le salarié dans la réalisation du bilan du stage, voire d'une éventuelle mobilité professionnelle.

Ce dispositif, mis en œuvre entre 1997 et 2001, partage ses principales caractéristiques avec les pratiques d' «accompagnement» (cf. par exemple les numéros que Travail et emploi et Informations sociales leur ont consacré en 2009 et 2012) développées à la même époque dans le cadre des politiques sociales et de l'emploi, politiques publiques dites d' «activation» des chômeurs (DivaY, 1999), des salariés licenciés en cellule de reclassement (MAZADE, 2005), mais aussi des allocataires de minima sociaux (Duvoux, 2009) ou des populations défavorisées (FASSIN, 2004) : une rhétorique sur l'autonomie des individus et leur adaptation aux contraintes du marché du travail; une individualisation des outils et pratiques de gestion des populations; une incitation à interroger la situation d'emploi par un «travail sur soi» (VRancken, Macquet, 2006) et la convocation pour son interprétation d'outils d'inspiration psychologique (Stevens, 2008; Bresson, 2012). Entreprise de Soi se distingue néanmoins des politiques d'activation en se déroulant au sein même de l'entreprise et en s'adressant à une population composée à $86 \%$ d'ingénieurs et cadres de recherche et développement (R\&D), non pas en situation de chômage (Pochic, 2001a) mais en 
situation d'emploi, bénéficiant d'un haut niveau de qualification, d'un statut protecteur et d'une position économique et sociale élevée (voir encadré 1).

\section{Encadré 1}

\section{Une enquête ethnographique de trois ans dans l'entreprise Siris}

Les analyses présentées dans cet article sont issues d'une recherche doctorale fondée de manière centrale sur une enquête ethnographique de trois ans (1998-2001) dans une entreprise spécialisée dans la conception de grands systèmes informatiques. L'enquête s'est déroulée en grande partie dans la division consacrée à la conception de produits informatiques, dans le principal centre de recherche et développement (R\&D) de Siris en France. Aussi concerne-t-elle une population très qualifiée, composée à $86 \%$ d'ingénieurs et cadres. Cette population est également largement masculine (74 \%) et vieillissante $(63 \%$ des salariés enquêtés ont plus de 44 ans; $50 \%$ ont une ancienneté chez Siris supérieure à 20 ans).

L'enquête a pris comme objet central mais non exclusif le dispositif Entreprise de Soi et a mêlé observation (participante ou non), entretiens (biographiques, semi-directifs, souvent répétés) avec 79 salariés, et recueil de documents divers (archives publiques et privées, journaux internes et lettres d'information, tracts syndicaux, documents de travail, etc.). Elle a eu l'avantage de me mettre en contact avec des ingénieurs en cours de carrière et de bénéficier de ce contact de manière suffisamment durable pour suivre et approfondir l'analyse longitudinale de leur parcours et la variété de leurs comportements.

La recherche s'est également appuyée sur une enquête par questionnaire auprès de 320 salariés, représentatifs des 1864 salariés que comptait alors la division R\&D de Siris. Ce questionnaire biographique visait à recueillir rétrospectivement le parcours professionnel des individus interrogés, depuis la fin de leurs études jusqu'au moment de l'enquête (novembre 2000), en leur demandant de préciser leurs employeurs successifs, les différents statuts et fonctions occupés, leur échelon, leur lieu de travail, les raisons pour lesquelles ils ont été amenés à changer de poste, les formations importantes ou qualifiantes suivies; il comprenait également des questions sur leur rapport à l'emploi, leurs conditions de travail, leurs origines sociales, leur situation familiale et leurs activités hors-travail. Sur les 320 individus interrogés, 98 avaient participé à la formation Entreprise de Soi, ce qui a permis d'établir des comparaisons entre les deux souspopulations.

Qu'un dispositif s'adresse à cette population en ces termes peut a priori sembler étonnant : ce groupe professionnel n'est-il pas caractérisé par une marge d'autonomie importante, une implication morale induisant un «sens des responsabilités», une auto-administration des contraintes? Et, plus particulièrement pour les ingénieurs de R\&D, d'une disposition à l'innovation et au changement? La mise en œuvre d'une telle formation à destination de ce "salariat de confiance» (Bouffartigue, 2001a) révèle précisément sa déstabilisation (Bouffartigue, 2001b) : touchés de manière inédite par les restructurations sociales, menacés de déclassement ou de risque de chômage, ces cadres et ingénieurs sont sommés - comme des travailleurs ordinaires - d'adopter de nouveaux comportements d'emploi pour se convertir au modèle des «carrières nomades » (ZunE, 2002).

Pourtant, si l'objectif affiché d'Entreprise de Soi est de transformer les comportements des salariés, une observation longitudinale et en contexte montre une réalité moins univoque : les significations accordées au dispositif sont plurielles et témoignent des marges de manœuvre des différents acteurs de l'entreprise pour se l'approprier. Cette hétérogénéité est présente dès l'origine par ce qu'importent de leur trajectoire professionnelle et politique les trois concepteurs d'Entreprise de Soi. Elle se retrouve ensuite dans les motifs d'inscription des participants, dans les pratiques de formation proposées par les animateurs des stages et enfin dans les devenirs professionnels.

L'intérêt de l'enquête ethnographique sur laquelle repose cet article (voir encadré 1) est précisément d'avoir permis d'observer la vie du dispositif sur une longue période et d'assister aux différentes étapes de sa "carrière» au sein de l'entreprise et en dehors, mais aussi de suivre les devenirs des acteurs qui l'ont porté et s'en sont saisi. Aussi, cet article entend revenir de manière succincte sur chacun de ces moments d'Entreprise de Soi en montrant comment s'effectuent ces appropriations et comment s'articule cette pluralité de positions et de situations. Dans un premier temps, il analyse la construction de la formation comme le produit d'une alliance improbable entre trois acteurs aux trajectoires professionnelles et politiques antagonistes, ce qui conduit à lui donner un caractère profondément ambigu. Il explore ensuite comment des salariés interprètent Entreprise de Soi et s'en saisissent pour résoudre diverses tensions ou ruptures provoquées par les transformations de l'entreprise. Après quoi, il décrit les pratiques de « travail sur soi » mises en œuvre durant la formation et souligne leur ambivalence, en montrant tout d'abord des effets de réenchantement subjectif pour les participants, puis en mettant au jour des effets latents, largement impensés par les acteurs de la formation, de rapports sociaux de sexe et de classe. Ainsi, en replaçant la formation dans le processus de recomposition des normes d'emploi au sein de l'entreprise et du marché du travail, puis en mettant en relation les devenirs des participants après la formation avec leur trajectoire professionnelle antérieure et leurs propriétés sociales, l'article montre comment Entreprise de Soi recompose un 
ordre social qui impose de nouvelles catégories de pensée et d'action professionnelles et reproduit des inégalités sociales ${ }^{(3)}$.

\section{La conception d'Entreprise de Soi fondée sur une entente improbable}

En 1997, Siris développait une politique de gestion des compétences centrée sur la mobilité de ses salariés. Le contexte de l'entreprise était alors caractérisé par un fort déclin - l'effectif avait été réduit de plus de moitié en une décennie ${ }^{(4)}$ - et par la volonté de déplacer les salariés des «centres de coûts» que représentaient les centres de recherche et développement (R\&D) vers les «centres de profit» qui désignaient les services informatiques. La politique d'incitation à la mobilité était accompagnée d'un discours selon lequel l'entreprise ne pouvait plus garantir la stabilité des emplois de sorte que les salariés devaient rompre avec une «culture maternante pour s'adapter à une culture entrepreneuriale» $(5)$.

C'est pour soutenir cette ambition que Philippe, $\mathrm{DRH}$ de la division $\mathrm{R} \& \mathrm{D}$ de Siris, demandait à deux consultants internes en formation, Christian et Cécile, d'élaborer une formation visant à «encourager, favoriser, actionner la mobilité»( ${ }^{(6)}$. Le fruit de leur travail fut Entreprise de Soi, dont le titre est inspiré d'un essai du consultant en management Bob Aubrey (1994).

Les concepteurs du dispositif se retrouvaient pour soutenir et promouvoir la formation dans l'entreprise, mais aussi en dehors ${ }^{(7)}$. Tous trois tenaient un discours fondé sur les mêmes arguments : le monde du travail ferait face à une "nouvelle donne économique, sociale et technologique» qui impliquerait de changer de modèle de carrière et de remplacer "sécurité d'emploi» par «flexibilité», «promotion» par "autogestion de la carrière», et formation par «marché des compétences compétitives»; ces évolutions nécessiteraient un «changement de culture» où chaque salarié

(3) Je tiens à remercier Sarah Ghaffari, Séverine Misset, Fabienne Pavis et Marie Ponnet pour avoir organisé cette discussion scientifique sur les outils de gestion. Je les remercie également, ainsi que le comité de rédaction de Travail et emploi et les rapporteurs, pour leurs conseils et suggestions qui ont permis d'améliorer cet article, dont je porte seule la responsabilité.

(4) L'effectif mondial était alors passé de 45000 à 21000 salariés de 1988 à 1997 (sources : Bilans sociaux, Siris).

(5) Extraits d'une interview de Philippe, directeur des ressources humaines (DRH) de la division R\&D de Siris dans le journal interne d'entreprise, novembre 1996 (archives).

(6) Extrait de notes de réunion prises par Cécile (archives).

(7) Ils sont à l'initiative d'une association appelée Réseau et promotion d'Entreprise de Soi, regroupant quelques consultants, DRH, et salariés soucieux de diffuser le concept dans d'autres organisations, privées, publiques ou même associatives. deviendrait «plus mobile et plus autonome»(8). Entreprise de Soi est pensé comme une réponse à cet impératif de changement et permettrait de concilier les intérêts du salarié et ceux de l'entreprise : pour le salarié il s'agirait de mieux se connaître «pour augmenter la force et la clarté de [ses] choix» et pour devenir «acteur-auteur de son propre développement personnel et professionnel»; pour l'entreprise, il s'agirait d'avoir des collaborateurs davantage mobiles, «bien dans leur peau», porteurs d'initiatives, anticipant les changements du marché. De cette manière, les participants à Entreprise de Soi deviendraient des «vecteurs de changements» et permettraient à «la nouvelle culture» de se diffuser.

L'entente des concepteurs d'Entreprise de Soi, voire leur complicité, était pourtant loin d'être acquise. Il était de notoriété publique dans l'entreprise qu'ils avaient des convictions politiques fortement divergentes : Philippe y avait provoqué un certain scandale en se présentant sur une liste du Front national aux élections municipales de 1995, faisant éclater au grand jour son militantisme de jeunesse au GUD dans les années 1970; Christian avait mené une carrière syndicale très active à la CFDT et militait alors chez les Verts; enfin, Cécile avait été représentante du personnel et élue au comité d'établissement sous l'étiquette $\mathrm{CGT}^{\left({ }^{(9)}\right.}$.

Cette entente improbable a pu etre comprise par l'analyse de leurs carrières (Hughes, 1996) à la fois sociales, professionnelles et politiques ${ }^{(10)}$. Celle-ci a permis de montrer qu'Entreprise de Soi est le lieu de recyclage de savoirs et croyances construits lors de ces carrières, et permet à chacun de lui donner une signification particulière, conciliant dispositions et positions professionnelles, politiques et symboliques.

\section{Philippe, DRH, ancien militant Front national}

Ainsi, Philippe, issu d'une famille de la bourgeoisie parisienne, détenteur d'une maîtrise de droit des affaires obtenue en 1979 et travaillant depuis dans les ressources humaines, retrouve dans cette formation un appel à la flexibilité, à la responsabilité individuelle et à une appréciation marchande des situations professionnelles des salariés, qui correspondent à ses propres convictions et analyses

(8) Ces propos et ceux qui suivent sont extraits de la présentation d'Entreprise de Soi faite aux salariés de Siris R\&D en janvier 1999, par Philippe, Christian et Cécile (observation participante).

(9) GUD : Groupe union défense; CFDT : Confédération française démocratique du travail; CGT : Confédération générale du travail.

(10) Ces carrières, ainsi que les relations entre Philippe, Christian et Cécile, ont été retracées lors d'entretiens biographiques répétés et lors de la fréquentation régulière, si ce n'est quotidienne, de ces trois acteurs pendant les trois années de l'enquête ethnographique. Elles ne sont cependant ici que très brièvement évoquées. 
- libérales - du contexte de l'entreprise. Il dit à propos d'Entreprise de Soi :

"L'idée c'était de se dire: on n'est pas là uniquement pour donner un travail et du salaire, on est là aussi pour développer l'employabilité des gens, faire en sorte finalement que ces gens, le jour où il y a un problème dans l'entreprise, ou qu'ils ont un problème dans leur travail, eh bien que ça soit pas un drame parce qu'ils y seront préparés, ils seront formés, ils auront une valeur marchande. L'objectif c'était de pousser la population de [Siris] à se reposer la question de sa valeur marchande.»

\section{Christian, consultant en formation, ancien militant CFDT}

Christian a, à l'inverse de Philippe, été marqué par des espaces de socialisation politique situés à gauche : après un double cursus de droit public et de sciences politiques (IEP), il suit en 1967-1968 des études de sociologie à La Sorbonne contre l'avis de son père juriste. Il entame alors une carrière militante semblable à cette génération de militants de gauche façonnée par les mouvements catholiques et les mouvements étudiants, engagée dans les revendications politiques et syndicales de la gauche auto-gestionnaire (PSU, CFDT), vivant son activité professionnelle dans la formation continue comme un prolongement de ses positions politiques, et abandonnant au cours des années 1980 le terrain des grandes causes révolutionnaires pour développer de nouvelles formes d'engagement moins institutionnelles et plus locales (écologie, altermondialisme, etc.; Gaubert, 2005; Mauger, 1994). Christian poursuit ainsi dans Entreprise de Soi sa réflexion sur l'autonomie et l'auto-formation permanente; il y fait la démonstration de sa conversion politique puisqu'il croit désormais davantage à la nécessité de la transformation des dispositions individuelles plutôt qu'à celle des rapports sociaux. Il explique :

\begin{abstract}
«Dans Entreprise de Soi il y a fondamentalement une démarche auto. Ça, ça fait partie aussi de toute une réflexion politique sur la question de l'autonomie. La conquête de l'autonomie personnelle et l'autonomie de pensée m'apparaît être un acte décisif. [...] Entreprise de Soi ça a été un enjeu personnel important qui a été cette redéfinition par rapport au marxisme, par rapport au truc collectif étatiste, structure bureaucratique, etc. C'est un enjeu de changement social mais au niveau des micro relations. Je crois plus du tout à des grands changements de structures parce que c'est des mensonges absolus ça.»
\end{abstract}

\section{Cécile, consultante en formation, ancienne élue CGT}

Enfin, Cécile, diplômée de l'École polytechnique féminine en 1967, ingénieure informatique reconnue pendant près de trente ans et reconvertie dans la formation professionnelle continue en 1995 suite à une succession de réorganisations, importe - et trouve - dans Entreprise de Soi des convictions et savoir-faire issus de son expérience de sympathisante et d'élue CGT. Représentante du personnel (au CHSCT ${ }^{(11)}$ puis au comité d'établissement) lors d'une période de restructurations sociales, et alors qu'elle-même a subi à plusieurs reprises des suppressions de poste, elle a été amenée à privilégier le face-à-face direct aux négociations collectives et aider les salariés dans des situations de conflit, de menace sur l'emploi et de difficultés personnelles. Or c'est un travail similaire d'écoute et de réparation psychologique qu'elle retrouve dans Entreprise de Soi et qui fonde son engagement dans la formation ${ }^{(12)}$ :

«La genèse d'Entreprise de Soi c'est une demande de Philippe, et c'est une opportunité de mettre en place un parcours de développement personnel pensant que c'était une chose qui pouvait énormément aider les gens dans la situation dans laquelle ils se trouvaient à cette époque-là, après les plans sociaux.»

Alors que les positions politiques mais également professionnelles de ces trois acteurs les conduisaient à une situation conflictuelle(13), leur entente inédite autour de ce dispositif souligne combien Entreprise de Soi résulte d'une forme de compromis entre une pensée de droite libérale, une pensée de gauche autogestionnaire et une pensée de gauche altruiste. Ces systèmes de croyances, construits lors de carrières morales et militantes, ont été absorbés et sont présents dans le projet Entreprise de Soi. Si cette analyse montre l'importance des dynamiques politiques dans la production d'outils de gestion, et permet de saisir en situation la formation de ce que Luc Boltanski et Ėve Chiapello (1999) ont nommé le «nouvel esprit du capitalisme», elle permet également de comprendre en quoi Entreprise de Soi présente une ambiguïté forte entre, d'un côté, une volonté de conversion des dispositions des salariés de Siris et d'un autre, un dessein de réparation morale face aux crises auxquelles ils sont confrontés. Elle montre ainsi pour quelles raisons il peut être interprété de diverses manières, et explique pourquoi les principaux syndicats de Siris y sont

(11) CHSCT : Comité d'hygiène, de sécurité et des conditions de travail.

(12) On retrouve là un processus similaire à celui que MarieHélène LECHIEN (2002) avait analysé à propos de l'évolution du rapport à l'action militante de certaines femmes syndicalistes engagées dans des activités humanitaires.

(13) Sans pouvoir présenter ici l'ensemble de la configuration de leurs relations, on peut tout de même préciser que, l'année précédant la création d'Entreprise de Soi, le service Formation auquel appartenaient Christian et Cécile avait été entièrement restructuré par Philippe, conduisant à des suppressions de postes et des mobilités contraintes, ainsi qu'au déclassement de Christian qui s'était vu retirer la responsabilité du service. 
opposés ${ }^{(14)}$, mais aussi pourquoi et comment les salariés qui décident de s'y inscrire se l'approprient selon des modalités plurielles.

\section{Encadré 2}

\section{Proposer une analyse sociologique des inscriptions à Entreprise de Soi}

Comprendre les raisons de l'inscription de salariés de Siris à Entreprise de Soi nécessitait dans un premier temps de mettre à distance le registre d'inspiration psychologique spontanément mobilisé par les participants pour justifier leur démarche ( "réfléchir sur moi-même», "mieux me connaître», "cesser de fuir les conflits", "m'aider à modifier mon comportement», "mieux gérer mon stress», "retrouver confiance en moi», "reconstruire ma personnalité », etc.). Un principe central de recherche a permis cette distanciation : celui de considérer l'ancrage d'Entreprise de Soi dans les temporalités individuelles et organisationnelles ${ }^{(1)}$, ce qui conduisait à adopter une démarche méthodologique triple : tout d'abord effectuer un travail important d'objectivation, par la reconstitution des parcours professionnels des participants afin de repérer la suite des positions professionnelles occupées (statut, activité de travail, position dans la grille de classification, relation avec l'équipe de travail, lieu de travail, etc.), leurs dynamiques et leurs ruptures (pente, longueur, forme de la trajectoire professionnelle et sociale). Ce travail a été réalisé par l'analyse des entretiens biographiques, parfois répétés, avec les participants à Entreprise de Soi $(n=43)$ et de leurs réponses au questionnaire biographique (voir encadré $1 ; n=98$ ). Ici, l'appui sur des éléments objectivables a été d'autant plus nécessaire que les enquêtés, passés par Entreprise de Soi, y avaient appris - comme l'article le montrera ultérieurement - à présenter leur trajectoire de manière à lui donner une cohérence et

(1) Suivant par là les analyses de Claude Dubar (1984, p. 94) : «la question des motivations à se former des stagiaires de formation continue (...) renvoie aux trajectoires sociales, professionnelles et culturelles des stagiaires et aux significations et attentes investies dans la formation pour modifier, infléchir ou consolider cette trajectoire. (...) Toute motivation à se former résulte d'une articulation d'exigences "externes" liées à une situation et d'aspirations "internes" résultantes d'une trajectoire, d'incitation "objectives" de l'environnement et d'espoirs "subjectifs" de la personnalité». à la valoriser. Si ces entretiens comportaient ainsi le risque d'une reconstruction a posteriori du sens donné à leur trajectoire, ils contenaient également de nombreuses expressions véhiculées durant la formation, et pouvaient, par moments, refléter une posture réflexive singulière, fidèle aux recommandations des formateurs d' 'être sincère et bienveillant avec soi-même". Comme Muriel Darmon (2007) qui, enquêtant auprès de jeunes filles anorexiques et socialisées aux entretiens cliniques lors de leur hospitalisation, constate que certaines d'entre elles «sont devenues des quasi-professionnelles du discours sur soi» (p. 46), les entretiens réalisés avec les participants à Entreprise de Soi portent "la marque de la redéfinition de soi» (p. 215) opérée pendant la formation, marque que je pouvais d'autant plus repérer que j'avais moi-même participé aux séminaires et avais pu observer ce travail de redéfinition de soi par les catégories et pratiques mises en œuvre lors d'Entreprise de Soi. Afin de soutenir ce travail d'objectivation, un second principe a été appliqué : celui de la comparaison de leurs discours avec ceux des autres salariés. Si leurs trajectoires professionnelles ne sont pas particulièrement différentes de celles de leurs collègues, en revanche lors des entretiens biographiques réalisés, on repère chez eux une disposition à accorder crédit plus rapidement à la démarche et aux questions que (se) pose la chercheuse en sciences sociales, à accepter de jouer le jeu de l'entretien, mais aussi une plus grande propension à faire preuve de réflexivité, à raconter leur carrière et leur expérience avec précision. Enfin, ces principes de recherche ont systématiquement été accompagnés d'un travail de contextualisation, et d'articulation des trajectoires aux contextes socio-historiques et organisationnels dans lesquels elles se déroulaient, grâce à une reconstitution de l'histoire de l'entreprise et de ses politiques industrielles et de gestion du personnel.

Au final, il s'agissait bien de considérer l'inscription à Entreprise de Soi comme un processus, de la replacer dans des temporalités longues, celles des carrières individuelles, elles-mêmes définies par les temporalités de l'entreprise et celles des transformations sociales. Comme l'a notamment fait Sophie Denave (2006, p. 109) à propos des ruptures professionnelles, une telle démarche de recherche revenait à «traiter ensemble les contextes de crise propices aux changements, les événements déclencheurs du passage à l'acte, et les formes différenciées prises par les désengagements et les engagements professionnels".

(14) Dès les premières communications sur Entreprise de Soi dans l'entreprise, les syndicats expriment leurs inquiétudes : la CFDT l'interprète comme un moyen pour que «les salariés acceptent leur départ, de préférence hors de la division $R \& D$, et encore mieux de Siris» et la CGT dénonce «un plan social déguisé» qui de surcroît «masque les réalités du marché du travail». Les deux syndicats critiquent également l'individualisation qu'ils voient à l'œuvre dans le dispositif et qu'ils analysent comme une «déstabilisation des communautés de travail» et une responsabilisation des salariés, parallèle à un désengagement de l'entreprise. Enfin, ils soulignent le risque de stigmatisation de salariés déjà fragilisés par un contexte d'emploi tendu, et celui d'instrumentalisation d'éléments de leur vie hors-travail dévoilés lors des séminaires de « développement personnel» (sources : extraits de tracts syndicaux et d'entretiens avec les représentants syndicaux). 


\section{Des appropriations plurielles, ancrées dans les transformations de l'entreprise}

En cinq ans (1997-2001), parmi les quelque 1800 salariés que compte la division R\&D de Siris, 160 décident de participer à Entreprise de Soi ${ }^{(15)}$.

Lors des entretiens ou des échanges plus informels avec ces derniers, revenait très souvent l'idée selon laquelle Entreprise de Soi était, pour eux, «arrivé au bon moment». La récurrence de ces propos indiquait clairementl'ancrage du dispositifdans unetemporalité biographique. Il importait dès lors de recontextualiser les raisons évoquées pour l'inscription à Entreprise de Soi dans les trajectoires des salariés, elles-mêmes replacées dans les cadres sociaux dans lequel elles s'inscrivaient (voir encadré 2).

Aussi, en reconstituant les parcours des participants, de manière à la fois objective et subjective, il était possible de constater que l'inscription à la formation suivait systématiquement une rupture biographique, un «turning point» (Hughes, 1996, pp. 59-136), interrompant une dynamique professionnelle, bouleversant des «univers de croyances » (Dubar, Demazière, 1997, p. 99), perturbant des «ordres négociés» (STRAuSs, 1992, p. 87-112). Sources de multiples décalages, de «désajustements entre l'incorporé et la situation nouvelle» (LAHIRE, 1998, p. 57), les effets de ces événements biographiques se traduisaient par des sentiments de fatigue, de doute, d'impuissance, de menace ou de pression mentale, et déclenchaient des pratiques de réflexivité. Ce sont ces pratiques individuelles que rencontre l'offre institutionnalisée de «travail sur soi» proposée par Entreprise de Soi. Suivant une démarche idéale-typique ${ }^{(16)}$, quatre grandes positions professionnelles, à la fois objectives et subjectives, déclenchant l'inscription ont pu être distinguées.

(15) L'analyse des propriétés sociales mais aussi des trajectoires professionnelles des participants montre qu'ils se distinguent peu de leurs collègues en termes d'origine sociale, de niveau de qualification, d'échelon, d'âge et d'ancienneté (comparaison conduite grâce à l'enquête par questionnaire, $c f$. encadré 1). Concernant ces variables, une seule différence notable les distingue : on retrouve davantage de femmes parmi les participants (39\%) que dans la population d'origine $(26 \%)$. Ceci s'explique à la fois par un plus grand intérêt féminin pour la culture psychologique, construit sur une division sexuelle du travail entre «monde des choses humaines» et «monde des choses matérielles» (Mauger, Poliak, 2000; Bourdieu, 1990), mais aussi par un contexte de travail et d'emploi qui fragilise particulièrement leurs positions professionnelles (STEVENS, 2007).

(16) Cette démarche repose sur l'analyse approfondie, inspirée de la méthodologie proposée par Didier DEMAZIÈRE et Claude Dubar (1997), des entretiens biographiques avec les participants d'Entreprise de Soi, et croisée avec une analyse qualitative et quantitative de leurs réponses au questionnaire biographique (voir encadré 1).

\section{S'inscrire à Entreprise de Soi suite à une perte de statut professionnel (parcours-type I)}

Certains salariés viennent à Entreprise de Soi à la suite d'une perte de statut professionnel. Ils ont connu récemment un déclassement (confiscation d'une responsabilité hiérarchique ou technique) ou sont directement confrontés à une menace de suppression de leur poste de travail. Affectés par cet événement, ils voient dans Entreprise de Soi un support de réparation morale (beaucoup témoignent du besoin de reconstruire une image positive d'euxmêmes, rendue négative par leur mise à l'écart) et un moyen de se reclasser.

\section{Suite à une non-reconnaissance professionnelle (parcours-type II)}

D'autres salariés rejoignent le dispositif en réaction à une expérience de non-reconnaissance professionnelle, celle-ci pouvant se manifester par une opposition de leur hiérarchie à leurs vœux de promotion, ou par une mobilité qui leur a été imposée malgré leurs besoins de stabilité (pour maîtriser une technologie, un poste de travail, pour des raisons familiales, etc.). Ils cherchent alors dans Entreprise de Soi des ressources pour se changer eux-mêmes, afin de mieux correspondre aux nouvelles exigences de carrière de l'entreprise et ainsi espérer relancer une trajectoire bloquée ou déstabilisée.

\section{Suite à une intensification du travail (parcours-type III)}

D'autres encore font face à une situation d'intensification du travail due au contexte de restrictions des emplois, de raccourcissement des délais de production, de mobilités imposées ou d'individualisation des outils de gestion de la main-d'œuvre (Gollac, Volkoff, 1996). Certains salariés confrontés à des formes de harcèlement moral, de stress, de dépression, de conflits interpersonnels ont recours au dispositif pour apaiser une souffrance psychique et définir une sortie de crise.

\section{Suite à un événement familial marquant (parcours-type IV)}

Enfin, un dernier groupe de salariés s'inscrit à Entreprise de Soi pour des préoccupations qui semblent a priori éloignées du monde du travail, puisque leur participation se déroule après un événement familial marquant (naissance, décès, maladie, divorce, conflits avec les enfants adolescents). Ces participations révèlent de manière significative la possibilité de s'approprier Entreprise de Soi de manière relativement libre, en cherchant à l'utiliser pour résoudre des questions d'ordre privé. Pourtant, chez ces participants, la question du travail n'est pas absente, loin de là. Elle est même posée de manière centrale, par la place qu'il occupe dans une existence dont le sens a été bouleversé par la mort, la 
séparation ou la difficile transmission intergénérationnelle. C'est alors une réflexion sur le sens de leur travail que ces salariés engagent. Leurs entretiens témoignent tous du sentiment d'avoir perdu la maîtrise de leur activité de conception informatique, à la fois dans son caractère matérialisé et dans la recherche constante de perfectionnement. Sous les effets conjugués de changements technologiques, de décisions stratégiques et de réorganisation de l'activité de $\mathrm{R} \& \mathrm{D}$, ces ingénieurs et techniciens disent leur profonde insatisfaction à intervenir sur une «machine sur laquelle on ne peut plus bidouiller», dont ils ne connaissent pas le fonctionnement des composants - pour l'essentiel achetés désormais à d'autres constructeurs informatiques -, et qui est de plus en plus rapidement obsolète (STEVENS, 2010). Leur travail qu'ils concevaient comme une activité de création, définie par des exigences d'innovation, de performance mais aussi de «beauté technique», validées par leurs pairs, est bousculé par des logiques de rentabilité à court terme et des impératifs de productivité. Le rapport au temps, à la pérennité de leurs actes, au sens même de leur existence, qui était engagé à travers leur travail est ainsi bouleversé. Entreprise de Soi est dans ce cas investi comme un espace pour mener une réflexion sur le sens du travail et élargir le champ de leurs possibles professionnels.

\section{Des inscriptions qui s'ancrent dans les ruptures de la vie de l'entreprise}

À travers ces rapides descriptions des logiques d'accès à Entreprise de Soi, on perçoit aisément comment elles s'ancrent dans les ruptures que l'entreprise Siris a connues dans les années 1990 : réduction importante de la main-d'œuvre, redéfinitions des modèles d'emploi et de gestion des carrières, changements du modèle d'organisation du travail et des formes de mobilisation de la maind'œuvre, transformations de l'activité de travail des informaticiens. Les salariés de Siris sont confrontés à des situations de blocage professionnel, de mobilités imposées, d'intensification du travail, d'altérations de leur activité de travail, et doivent envisager un changement alors même que leurs possibilités objectives de mobilité diminuent. Ayant le sentiment d'être dans une impasse, ils se sentent prêts à accorder de l'intérêt à ce dispositif qui les invite à reconsidérer sous un nouveau jour leur situation.

Dans un tel processus, les attentes à l'égard d'Entreprise de Soi ne sont pourtant pas identiques les unes aux autres. Certaines sont d'ailleurs perçues comme moins légitimes que d'autres - comme celle de saisir le programme comme une forme de psychothérapie à la suite d'une déstabilisation personnelle - et peuvent être rendues clandestines. Ces premières appropriations, diversifiées, se poursuivent au cours du dispositif.

\section{Des pratiques de réenchantement subjectif...}

Les principes d'inscription volontaire et de confidentialité, la diversité des thèmes et des approches proposés et les méthodologies dites non-directives, permettent aux salariés de trouver des éléments de réponse aux interrogations qui les ont poussés vers Entreprise de Soi.

\section{Une requalification symbolique}

Ainsi les salariés confrontés à une perte de statut professionnel (parcours-type I) sont amenés à plusieurs reprises à raconter leur parcours et à y repérer les éléments satisfaisants et constructifs. La consigne formulée par l'ensemble des consultants-animateurs des séminaires est toujours la même : parler de soi en termes positifs, faire référence à des situations de réussite et non d'échec, mettre en avant ses qualités plutôt que ses défauts, aborder les difficultés rencontrées en présentant les «enseignements personnels» qu'elles ont permis de tirer. La trajectoire passée est considérée comme un réservoir d'expériences dans lequel le participant à Entreprise de Soi doit apprendre à puiser des éléments témoignant de ses capacités et de ses savoir-faire. Par exemple dans le séminaire «Moi et ma façon d'agir », il doit repérer «les actions ou les réalisations qui lui restent comme des moments plus marquants où il a eu la sensation de donner le meilleur de lui»; ou plus encore dans le séminaire "Moi et mon scénario de succès», il doit décrire de manière détaillée «trois expériences passées où il a particulièrement bien réussi».

L'intention de valorisation personnelle est présente à chaque instant du dispositif, y compris dans les consignes d' «écoute bienveillante» et de «non jugement» des participants entre eux. C'est d'ailleurs ce qui ressort le plus des évaluations des salariés qui ont suivi Entreprise de Soi : alors qu'ils étaient fragilisés par une situation professionnelle incertaine, une grande majorité déclare spontanément ressentir une plus grande «confiance en soi» à l'issue de la formation et expriment un sentiment de réassurance sur leurs capacités. C'est là tout le travail de «requalification symbolique» (MiLlet, ThIN, 2012, p. 68) réalisé au cours d'Entreprise de Soi.

Lors des différentes activités de récit de soi, les stagiaires développent également des compétences langagières spécifiques : puisant dans un répertoire de situations-types et de vocabulaire pour décrire et interpréter des situations passées, on repère chez eux une grande propension à raconter leur carrière, à en distinguer les points saillants, les périodes fastes, les contextes d'apprentissage professionnel, et au-delà, à dire, avec conviction, ce qui les caractérise, ce qu'ils savent faire ou pas, et ce qu'ils apprécient ou 
non. Ces compétences sont mises au service d'une stratégie narrative : bien que leur trajectoire soit marquée par les ruptures, ils parviennent parfois à en reconstruire une cohérence a posteriori, à la fois pour eux-mêmes et pour autrui, dans le but d'assurer leur reclassement professionnel.

\section{Un apprentissage des nouvelles règles de carrière}

Pour les salariés bloqués dans leur souhait de promotion ou tenus malgré eux de changer de poste de travail (parcours-type II), l'enjeu est $\mathrm{d}$ 'apprendre les nouvelles règles de carrière afin de s'y conformer ou d'en jouer pour tenter d'obtenir l'emploi souhaité. Ils saisissent dans Entreprise de Soi tout ce qui leur permet de "résoudre [leurs] problèmes de communication», ou d' «améliorer [leurs] comportements» qu'ils jugent inadéquats ou insuffisants et qui expliqueraient leur situation de non-reconnaissance professionnelle. Et de fait, Entreprise de Soi constitue - tout comme les formes d'accompagnement à la recherche d'emploi pour les cadres chômeurs analysées par Sophie PochIC (2001a, p. 2) - «un espace de socialisation [aux] nouvelles normes d'emploi fondées sur la logique employabilité».

C'est ainsi qu'ils découvrent dans la formation des explicitations mais aussi des mises en scène répétées de ce qu'est le «nouvel esprit de l'entreprise». Ils le trouvent bien sûr dans les nombreuses présentations du dispositif, mais plus fondamentalement, dans le langage utilisé pour parler de leur situation professionnelle : il s'agit d'un langage psychologique qui désigne l'individu et sa «psyché» comme le lieu de résolution des difficultés rencontrées, et qui explique que les participants affirment avec ces termes chercher à combler une «insuffisance», à «éliminer» ou «lever certains blocages», «travailler sur [leur] personnalité», «modifier [leur] comportement», "améliorer [leur] manière d'être», changer leur «mode de fonctionnement», réactiver leur «désir». Ce langage psychologique est doublé du langage de «la cité par projets» que décrivent BOLTANSKI et Chiapello (1999) : lors de différents exercices, les participants sont invités à décrire le «réseau» sur lequel ils peuvent compter, à définir les «valeurs» qui sont supposées guider leur action, à découvrir leur «talent $»$, etc.

Ce double langage est également incarné par les consultants-animateurs : la plupart présentent leur trajectoire en insistant sur la rupture qu'ils ont opérée avec le salariat pour devenir consultant indépendant afin de mener une activité leur permettent de «se réaliser». De la même manière, les "conseillers en stratégie de vie» que les participants peuvent rencontrer lors d'entretiens individuels, sont présentés comme des "personnalités qui ont affronté les événements de manière positive, ont osé les changements de vie, ont lancé des projets innovants, se sont adaptées aux conditions en cours. 》

Enfin, le «nouvel esprit de l'entreprise» est mis en scène. À travers divers exercices de mises en situation, les participants sont invités à ajuster leurs comportements aux bonnes règles de sociabilité professionnelle, c'est-à-dire à adopter une attitude relationnelle conciliante. S'appuyant sur des tests de personnalité, chaque participant découvre son «profil-type», puis apprend à reconnaître celui de son interlocuteur et à faire dialoguer au mieux ces deux profils, grâce à des schémas de communication préétablis. Ou bien, aidés par diverses techniques d'écoute et de reformulation, par un vocabulaire et des astuces pour exprimer leurs "émotions», les participants expérimentent, par des jeux de rôles et d'improvisation, des modes de relations dans lesquels ils doivent à la fois «rester [eux]même[s]» et "s'adapter à l'autre». Ou encore, dans un séminaire de "relooking», les stagiaires corrigent leur tenue vestimentaire, leur coiffure ou leur maquillage et apprennent à maîtriser l'image qu'ils renvoient d'eux-mêmes.

\section{Une réparation psychologique}

Les salariés soumis à une intensification du travail (parcours-type III) trouvent dans Entreprise de Soi une occasion d'exprimer mais aussi de partager leurs difficultés, de faire le récit de pressions, de harcèlement, de décrire leurs symptômes de stress ou d'épuisement mental. Les règles de confidentialité et de bienveillance mutuelle, mais aussi les encouragements des animateurs autorisent l'expression d'une parole intime. Les salariés découvrent alors qu'ils ne sont pas seuls à connaître ces tensions et, de cette prise de conscience collective, peut naître parfois un renversement du sentiment de responsabilité des difficultés auxquelles ils sont confrontés : les participants désignent l'entreprise, accusée de mauvaise gestion, voire de maltraitance, et une certaine colère se ressent au sein du groupe de parole. Ils cherchent alors des ressources pour affronter la situation conflictuelle qu'ils vivent et s'opposer à l'organisation (autrement dit, aux acteurs qui la représentent). Plusieurs séminaires leur proposent des techniques d'«affirmation de soi» (ou «assertivité») par lesquelles ils sont incités à apprécier, valoriser et défendre leurs positions face à leur entourage professionnel; ils se familiarisent donc avec des techniques de communications pour refuser des décisions arbitraires, pour négocier des charges de travail trop lourdes et pour apaiser les conflits. Ils sont ainsi légitimés dans un droit à «s'imposer», à «oser», à "se sentir capable», à «dire non», à «refuser», autant de termes utilisés dans les séminaires et repris par les participants enquêtés, et qui témoignent des négociations envisagées ou entreprises. 


\section{Une ouverture des possibles}

Enfin, les salariés perturbés par un événement familial (parcours-type IV) s'approprient Entreprise de Soi comme un espace d'interrogation de leur existence et, plus particulièrement, de la place qu'y occupe le travail, et cherchent ainsi un moyen de "se réaliser», "laisser une trace», et de «donner un sens». Ils y sont encouragés par le discours des concepteurs et des animateurs des séminaires qui les invitent à «ne plus séparer vie professionnelle et vie personnelle», mais à construire une cohérence individuelle entre l'ensemble de leurs activités professionnelles, familiales, associatives, etc., justifiant cette invitation par la nécessité de multiplier les supports identitaires face aux risques contemporains de précarité des emplois et de fragilité des liens affectifs.

Entreprise de Soi propose dès lors tout un travail de mise à distance de la vie de l'entreprise et d'ouverture du champ des possibles professionnels, qui ne laisse pas insensibles ces salariés insatisfaits et inquiets de leur situation actuelle. Un tel travail se réalise à de nombreuses occasions : lorsque les récits d'expérience doivent comporter des récits d'activités extra-professionnelles, valorisant particulièrement les activités d'expression (artistiques, sportives, etc.) ou celles tournées vers la relation à autrui (bénévolat, entraide, etc.), et explicitant les compétences acquises à ces occasions; ou lorsqu'un exercice consistant à "imaginer six actions que vous engageriez si vous aviez gagné un gros lot au loto», ou qu'un autre demandant d'exprimer «une passion, un rêve d'enfant que vous souhaiteriez réaliser $》$, incitent les salariés à s'extraire des contraintes financières et matérielles; ou enfin, lorsque des séminaires s'organisent autour de la définition de caractéristiques personnelles, qu'elles soient appelées «personnalité», «valeur», «mode de fonctionnement », «façon d'agir», ou «identité», mais qui toutes sont désignées comme à l'origine des attitudes, des choix et des positions de chacun, aussi bien au travail que dans la vie extra-professionnelle.

Par ces différents discours et exercices, les participants à Entreprise de Soi trouvent des éléments pour, dans une dialectique entre ipséité et mêmeté (RICEUR, 1990), construire une certaine cohérence biographique entre les différentes périodes et sphères sociales de leur vie, qu'ils parviennent parfois à traduire dans un nouveau projet professionnel, dans l'entreprise ou en dehors, dans l'informatique ou dans un autre secteur d'activité.

Les quatre types de pratiques rencontrés lors de l'observation participante d'Entreprise de Soi témoignent ainsi de deux processus : le premier est bien celui de l'appropriation par les salariés $\mathrm{du}$ dispositif à des fins variées, pour répondre à diverses inquiétudes provoquées par les transformations de l'entreprise. Le second est celui d'une acquisition de ressources pour faire face à des difficultés individuelles : acquisition d'un langage pour parler de soi, restaurer une image positive, réparer des souffrances psychiques; apprentissage des nouvelles règles du jeu professionnel pour pouvoir en jouer; expérimentation de techniques pour s'opposer ou résister à des pressions diverses; application d'outils réflexifs pour retrouver un sens à son activité professionnelle.

Présentées ainsi, les pratiques d'Entreprise de Soi ne semblent pas ambiguës : les salariés qui s'y sont inscrits semblent en sortir plus armés et, comme en témoignent leurs avis très positifs voire enthousiastes lorsqu'on les interroge directement à la suite de leur participation, Entreprise de Soi a bien un effet qu'on peut qualifier de réenchantement subjectif. Pourtant, cet effet n'est pas dénué d'une face plus sombre, révélée par l'objectivation des effets du dispositif sur les rapports sociaux, mais également par l'analyse des devenirs des salariés, quelques mois ou années après leur participation, après qu'ils ont cherché à mettre en pratique, en situation de travail, les enseignements qu'ils avaient pu tirer d'Entreprise de Soi.

\section{... qui masquent les rapports sociaux}

Si chacune des pratiques de «travail sur soi» proposées au cours de la formation peut fournir des ressources pour les participants, aucune en revanche ne change les conditions objectives de travail et d'emploi, et toutes ont des effets moins immédiatement manifestes sur les rapports sociaux. Une ambivalence du dispositif se dévoile.

\section{Une normalisation et une instrumentalisation de la réflexivité}

Certes Entreprise de Soi peut être un espace où les salariés trouvent les ressources langagières pour voir et présenter sous un meilleur jour leur trajectoire passée et envisager une mobilité promotionnelle. Mais si ces mises en récit et en scène de soi ont des effets de requalification symbolique, elles ont également des conséquences en termes de normalisation et d'instrumentalisation de la réflexivité. Celle que propose Entreprise de Soi est ainsi tournée vers l'adaptation aux nouvelles normes d'emploi, réduites à leurs dimensions comportementales et relationnelles. Comme le souligne Alain Eraly (1994, p. 142), en développant les «technologies de soi», le management contemporain est caractérisé par le fait que «la saisie de pans entiers de la vie humaine jusqu'alors abandonnés à la spontanéité de la vie affective et l'irréflexion des habitus [soit] bien moins orientée vers la découverte de soi que vers l'action sur soi. D'emblée la connaissance se veut instrumentale, elle est une méthode et sa 
validité est d'abord pragmatique». En témoigne par exemple ici l'utilisation du vocabulaire de la vente et du marché pour parler de soi (17).

\section{Un renforcement de l'auto-contrôle}

De même, l'apprentissage des nouvelles règles de carrière est ambivalent. On a pu constater qu'il constituait une forme de rattrapage pour tous les salariés de milieu populaire, qui n'étaient pas passés par les écoles d'ingénieur ou de commerce, et qui n'avaient pas intériorisé les normes d'emploi des cadres et ingénieurs; il permet ainsi aux salariés les moins dotés socialement et scolairement de chercher à s'y conformer au mieux en vue d'une reconnaissance professionnelle. Dans le même temps, il donne à d'autres salariés les outils nécessaires pour les maintenir à distance, voire pour en jouer par des adhésions de façade, révélant au passage un détournement des intentions $\mathrm{du}$ dispositif. Mais cet apprentissage conduit également à un renforcement de l'auto-contrôle : les difficultés professionnelles sont expliquées par des insuffisances psychologiques qu'un «travail sur soi » permettrait de corriger et chacun est tenu, quelle que soit la situation, de présenter une image de soi dynamique, agréable, conciliante et d'entretenir des relations pacifiées avec ses partenaires de travail (BALAzS, FAGUER, 1996).

\section{Une accentuation de l'individualisation, une négation des rapports sociaux}

Le processus est proche pour ce qui est des méthodes de réparation des souffrances psychiques : il ne s'agit en effet pas d'agir sur les causes structurelles et organisationnelles d'intensification du travail, mais bien de demander aux individus de s'adapter à leurs conditions de travail, de prendre en charge les tensions et les difficultés qui en résultent. Le séminaire «Moi et la gestion du stress» est typique de ce phénomène : il propose des techniques, «mentales, physiques et comportementales de réduction de stress» (relaxation, sophrologie, biofeedback, etc.) pour permettre à chaque participant de faire face aux pressions. Ici comme ailleurs, l'idée sous-jacente est que le salarié est impuissant à changer la situation professionnelle qui s'impose à lui, et plus largement l'ordre du monde. Entreprise de Soi contient ainsi des appels répétés à «accepter

(17) Ce vocabulaire - et le souci dont il témoigne - est très présent dans les entretiens: "Je me vends pas bien, je suis un peu timide» (Sophie). "Sur le marché, je pense que je pourrais me vendre avec cette étiquette-là» (Nadège). "Ma démarche d'aller vers les services c'est aussi de composer sur des techniques qui puissent permettre de me vendre à l'extérieur» (Bertrand). "Pour l'instant je me dis que j'acquiers encore des compétences qui me permettront de mieux me vendre sur le marché» (Céline). "J'arriverai peut-être mieux à me vendre. Parce qu'il y a des endroits où on n'a pas forcément besoin de technique pour faire quelque chose d'utile et d'intéressant.» (Guy), etc. le changement» et propose même pour cela des ateliers centrés sur le «travail de deuil» présenté comme nécessaire pour abandonner des positions professionnelles ou des valeurs jugées obsolètes et inadéquates. Dans d'autres séminaires, c'est l'expression «lâcher prise» qui fait florès et qui, là encore, invite à ne pas chercher à agir sur des phénomènes qui semblent immuables. C'est donc à l'individu, pensé de manière isolé, de changer, de s'adapter. Même lorsque les animateurs forment les participants à «dire non» pour s'opposer à des décisions arbitraires, ils rappellent l'exigence de paix sociale et délégitiment toute forme de conflictualité - interpersonnelle, a fortiori collective. Ainsi, ils indiquent le comportement adéquat - ne pas avoir d'attitudes agressives, formuler des propositions "gagnant / gagnant», etc. - pour négocier des charges de travail trop lourdes, refuser des injonctions, solliciter un changement. Par Entreprise de Soi, les processus d'individualisation - et de responsabilisation individuelle - mais aussi de dépolitisation à l'œuvre dans le monde du travail sont clairement renforcés, présentant alors paradoxalement le risque d'accentuer les souffrances que le dispositif prétendait apaiser ${ }^{(18)}$.

\section{Une reproduction de l'ordre social}

Enfin, l'ouverture du champ des possibles n'est pas aussi émancipatrice qu'elle le paraît au premier abord. Parce qu'elle repose sur une approche psychologisante, cette intention ignore complètement les processus de socialisation, inscrits dans des rapports sociaux - qui ont construit les dispositions des participants à Entreprise de Soi. Lorsqu'il s'agit de mobiliser et valoriser des expériences situées en dehors du travail, personne n'interroge le fait que les femmes mettent en avant des activités de soutien scolaire et d'éducation, d'expression artistique ou de bénévolat, qu'elles traduisent en compétences d'écoute, d'empathie ou d'altruisme; et qu'à l'inverse, les hommes valorisent des savoir-faire techniques acquis lors de la construction de leur maison ou d'activités de bricolage, des capacités de coordination éprouvées lors d'encadrement sportif, ou un sens des responsabilités mis en œuvre dans un engagement municipal. Sans réflexion sur la socialisation différenciée et la division sexuée du travail, ces compétences ou dispositions sont naturalisées et conduisent à la définition de nouveaux projets professionnels marqués par les appartenances de

(18) Il convient de souligner que ce processus de dépolitisation ou celui d'occultation du social présenté ci-après ne sont ni homogènes ni linéaires ou définitifs, mais sont l'objet de rapports de force qui peuvent, sous certaines conditions et à propos de certains phénomènes, les amener à être freinés ou inversés. C'est ce que montre Laïla SALAH-EDINE (2010) sur la catégorie du «harcèlement moral» qui a d'abord été analysée sous l'angle de la psychologisation du social avant de donner lieu à un traitement politique et juridique, conduisant à remettre au centre du débat la question de la dégradation des conditions de travail et la responsabilité de l'employeur. 
genre (Stevens, 2007). Il en est de même pour les salariés issus de milieu populaire ou les moins diplômés : leur champ des possibles s'avère modeste, voire restreint aux emplois de techniciens en SSII ${ }^{(19)}$, davantage soumis aux exigences de flexibilité et moins rémunérateurs. L'ordre social et sexué est ainsi reproduit.

\section{Les devenirs inégaux des salariés passés par Entreprise de Soi}

Or ce dernier constat a une portée plus large. Les effets de réenchantement d'Entreprise de Soi peuvent pour certains participants n'être que de courte durée. En effet, après avoir valorisé différents épisodes de leur trajectoire et explicité des compétences valorisables sur le marché du travail, après avoir expérimenté un certain nombre de techniques d'affirmation de soi ou de résistance aux pressions, après avoir défini un projet professionnel en accord avec leurs «valeurs», les participants retrouvent leurs situations et rythmes de travail de départ et cherchent à agir pour échapper ou remédier à leurs difficultés professionnelles (par exemple en sollicitant un changement de poste, en négociant leur temps ou charge de travail). Or tous n'y parviennent pas : seuls les mieux dotés y réussissent. C'est ainsi qu'on observant les positions professionnelles des salariés de Siris après leur participation à Entreprise de Soi, on peut distinguer quatre types de situation.

\section{Obtenir une requalification professionnelle au sein de Siris}

La première situation est celle de salariés qui parviennent à se saisir des outils proposés par Entreprise de Soi et à les transformer en ressources pour définir et négocier une nouvelle position, jugée plus qualifiante, dans l'entreprise Siris. Mais cette requalification chez Siris ne repose pas uniquement sur les ressources subjectives formalisées lors d'Entreprise de Soi; elle est déterminée par les ressources objectives. Ainsi cette issue concerne avant tout des personnes fortement diplômées, dont le parcours était antérieurement promotionnel. Elle touche plus particulièrement des salariés ayant une certaine ancienneté et qui, de ce fait, ont le capital social nécessaire au sein de l'entreprise pour négocier des promotions comme ils le faisaient auparavant ${ }^{(20)}$.

(19) SSII : Société de services en ingénierie informatique. (20) Cette conclusion rejoint là les analyses de Paul Bouffartigue et Sophie Pochic (2002) lorsqu'ils montrent que seule la fraction des cadres bénéficiant d'une position de force sur les marchés internes du travail parvient à s'approprier et à tirer profit de la rhétorique managériale sur les «cadres nomades».

\section{Obtenir une requalification professionnelle en quittant Siris}

La deuxième issue au sortir du dispositif est celle d'une requalification dans une autre entreprise. Là aussi, les participants parviennent à s'approprier dans Entreprise de Soi des ressources pour restaurer et revendiquer une nouvelle position qualifiante. Mais ils y ont également «fait le deuil» de leur carrière chez Siris, et développent un discours très critique à son égard et s'orientent vers une autre entreprise. Là encore, cette mobilité dépend de ressources objectives issues d'une trajectoire antérieure promotionnelle et concerne des salariés plus jeunes, dont les compétences acquises sont moins liées aux produits de Siris, et qui ont par conséquent une probabilité plus grande de s'insérer sur le marché externe.

\section{Un statu quo positif}

La troisième issue peut être qualifiée de statu $q u o$ positif. Il s'agit du cas de participants qui, après avoir suivi le dispositif, choisissent de ne pas changer de situation professionnelle parce qu'ils s'estiment dans l'impossibilité de négocier avantageusement une future mobilité; soit qu'ils jugent défavorable leur position vis-à-vis des employeurs, soit qu'ils considèrent comme trop coûteuse une adaptation aux nouvelles normes de carrière. S'ils parviennent à une telle conclusion, c'est parce que les offres qualifiantes chez Siris sont devenues rares, et ce d'autant plus qu'ils sont arrivés à un âge marqué institutionnellement par la mise à l'écart progressive. En effet, les participants concernés par cette issue sont tous âgés de plus de cinquante ans. Ils ont opté pour le maintien car ils ont intériorisé que les possibilités de mobilité leur sont objectivement défavorables. Ils valorisent néanmoins ce statu quo parce qu'il leur offre d'autres atouts jugés plus importants, comme la sécurité de l'emploi pour stabiliser leur fin de carrière, ou une certaine maîtrise de leur emploi du temps leur permettant la réalisation d'activités extraprofessionnelles. Ainsi, par l'intermédiaire d'Entreprise de Soi, ils se sont approprié l'idée d'un choix, délibéré et conscient, qui les amène à renoncer à leur ancien projet, à réenchanter d'une certaine manière leur position, et à négocier finement avec leur hiérarchie pour ne pas dévoiler leur jeu et risquer l'exclusion.

\section{Non reconnaissance et désillusion}

Enfin, la dernière position observée est celle de participants qui ne parviennent pas à restaurer, dans Siris ou ailleurs, une trajectoire professionnelle déstabilisée, qui ne disposent pas des ressources objectives nécessaires pour négocier une position requalifiante. Qu'ils aient ou non réussi à définir lors d'Entreprise de Soi, des éléments de valorisation de soi, tous sont confrontés à une 
non-reconnaissance de Siris et à une impossibilité de concevoir une insertion professionnelle satisfaisante. De retour dans leur situation de travail, ces salariés ne voient pas leurs conditions ou leur position changer. Ils restent dans une position déclassée, bloquée et conflictuelle; ils sont menacés d'exclusion alors qu'ils avaient caressé l'espoir de s'en sortir grâce à Entreprise de Soi. Pour ces participants, la crise qui les a menés à Entreprise de Soi n'est pas résolue et provoque une certaine désillusion. Cette dernière issue concerne des parcours dévalués sur le marché du travail, autrement dit, les «inemployables» que sont les salariés faiblement diplômés, âgés, ou peu mobiles, au parcours chaotique et difficilement valorisable sur le marché du travail. Elle révèle le hiatus majeur entre les objectifs de la formation Entreprise de Soi qui visent à faciliter la mobilité professionnelle, et les réponses qu'elle peut apporter qui sont d'ordre subjectif et intersubjectif, sans que les conditions structurelles ou organisationnelles du travail et de l'emploi ne soient interrogées ou modifiées. Les exercices de valorisation, de formation, de réparation ou d'émancipation des participants effectués durant la formation trouvent ici leurs limites : parce qu'ils sont réalisés en faisant largement abstraction des réalités professionnelles et du marché du travail, des rapports déséquilibrés entre employeur et salarié et des rapports sociaux, leur efficacité se révèle largement illusoire. Entre requalifications et réassignations sociales, l'enchantement subjectif que suscite Entreprise de Soi est au final inégalitaire parce qu'il réclame des ressources préalables inégalement réparties ${ }^{(21)}$.
$*$

$* \quad *$

Appréhender cet outil de mobilisation de la maind'œuvre qu'est Entreprise de Soi sous l'angle de l'ambivalence permet de rendre compte à la fois des dynamiques politiques qui le portent (entre une pensée de droite libérale et un militantisme de gauche converti à l'injonction des dispositions individuelles), des tensions professionnelles qui conduisent les salariés à s'y inscrire (entre restriction des emplois qui les oblige à envisager une mobilité, et transformations de l'entreprise et du marché du travail qui rendent bien difficiles les projections dans l'avenir), et des effets individuels et collectifs qu'il produit (entre dynamiques de réenchantement et de désenchantement, de requalification et de réassignation, d'émancipation et de reproduction sociales). En revanche, l'ambivalence n'est plus de mise lorsque sont finalement constatés des effets plus latents d'imposition de nouvelles catégories de pensée et d'action professionnelle qui renforcent la responsabilisation et l'auto-contrôle des salariés, contribuent à une dépolitisation des problèmes sociaux, soutiennent une psychologisation des rapports sociaux (CASTEL, 1981; VRANKEN, MAQUET, 2006; CAstel et al., 2008; Bresson, 2012) et légitiment l'entrepreneuriat comme nouveau modèle de régulation sociale. L'efficacité d'Entreprise de Soi tient finalement en cela : son caractère hétérogène permet des interprétations et des appropriations variées qui conduisent à une mobilisation et un engagement des différents acteurs en présence. L'esprit de l'outil en sort alors renforcé.

\section{Bibliographie}

Aubrey B. (1994), Le travail après la crise. Ce que chacun doit savoir pour gagner sa vie au XXI siècle, Paris, InterÉditions.

Balazs G., Faguer J.-P. (1996), «Une nouvelle forme de management : l'évaluation », Actes de la recherche en sciences sociales, $\mathrm{n}^{\circ} 114$, pp. 68-78.

Boltanski L., Chiapello E. (1999), Le nouvel esprit du capitalisme, Paris, Gallimard.

Bouffartigue P. (2001a), «Les métamorphoses d'un salariat de confiance : les cadres dans le tournant des années quatre-vingt-dix », Travail et emploi, $\mathrm{n}^{\circ}$ 86, pp. 107-125.
Bouffartigue P. (2001b), Les cadres. Fin d'une figure sociale, Paris, La Dispute.

Bouffartigue P., Pochic S. (2002), «Cadres nomades : mythe et réalités. À propos des recompositions des marchés du travail des cadres», Sociologia del lavoro, $\mathrm{n}^{\circ} 85$, pp. 96-106.

Bourdieu P. (1990), «La domination masculine», Actes de la recherche en sciences sociales, $\mathrm{n}^{\circ} 84$, pp. 2-31.

BRESSON M. (2012), «La psychologisation de l'intervention sociale : paradoxes et enjeux", Informations sociales, $\mathrm{n}^{\circ} 169$, pp. 68-75.

(21) Sophie Pochic (2001b) montrait également que la définition, sollicitée par les conseillers emploi de l'Apec (Association pour l'emploi des cadres), d'un projet professionnel par des cadres au chômage avait un faible effet sur le retour à l'emploi par rapport au poids des variables objectives que sont la durée du chômage, l'âge, le diplôme ou le sexe. 
Castel R., Enriquez E., Stevens H. (2008), «D’où vient la psychologisation des rapports sociaux?», Sociologies pratiques, $\mathrm{n}^{\circ} 17$, pp. 15-27.

Castel R. (1981), La gestion des risques. De l'antipsychiatrie à l'après-psychanalyse, Paris, Éditions de Minuit.

DARMON M. (2007), Devenir anorexique. Une approche sociologique, Paris, La Découverte, coll. «La Découverte poche. Sciences humaines et sociales », $\mathrm{n}^{\circ} 270$, [2003].

Demazière D., Dubar C. (1997), Analyser les entretiens biographiques. L'exemple des récits d'insertion, Paris, Nathan.

Denave S. (2006), «Les conditions individuelles et collectives des ruptures professionnelles », Cahiers internationaux de sociologie, vol. CXX, pp. 85-110.

Divay S. (1999), L'aide à la recherche d'emploi. Des conseils pour sauver la face, Paris, L'Harmattan.

Domingo P. (coord.) (2012), «L'accompagnement social vers l'emploi», Informations sociales, $\mathrm{n}^{\circ} 169$.

Dubar C. (1984), La formation professionnelle continue, Paris, La Découverte, coll. «Repères».

Duvoux N. (2009), L'autonomie des assistés. Sociologie des politiques d'insertion, Paris, Presses universitaires de France, coll. «Le lien social».

ERALY A. (1994), «L'usage de la psychologie dans le management : l'inflation de la "réflexivité professionnelle"», in Bouilloud J.-P., Lecuyer B.-P. (dir.), L'invention de la gestion. Histoire et pratiques, Paris, L'Harmattan, pp. 135-159.

Fassin D. (2004), Des maux indicibles. Sociologie des lieux d'écoute, Paris, La Découverte, coll. «Alternatives sociales ».

GAUBERT C. (2005), «Révolution culturelle et production d'un "intellectuel de proposition" (Pierre Rosanvallon)», in Tissot S. (dir.), Reconversions militantes, Limoges, Presses universitaires de Limoges, pp. 231-268.

Gollac M., Volkoff S. (1996), «Citius, altius, fortius. L'intensification du travail», Actes de la recherche en sciences sociales, $\mathrm{n}^{\circ} 114$, pp. 54-67.

Goux D. (coord.) (2009), «Accompagnement dans et accompagnement vers l'emploi», Travail et emploi, $\mathrm{n}^{\circ} 119$.

Hughes E.C. (1996), Le regard sociologique, Paris, Éditions de l'EHESS.

LAHIRE B. (1998), L'homme pluriel. Les ressorts de l'action, Paris, Nathan.

LECHIEN M.-H. (2002), «Aider les autres : trajectoires professionnelles, reconversions militantes et valorisation de la "personne"», in Collovald A. (dir.), L'humanitaire ou le management des dévouements. Enquête sur le militantisme de «solidarité internationale » en faveur $d u$ Tiers-Monde, Rennes, Presses universitaires de Rennes, pp. 79-132.

Mauger G. (1994), «Gauchisme, contre-culture et néolibéralisme : pour une histoire de la "génération de mai 68 " », in Chevallier J. (dir.), L'identité politique en question, Paris, Presses universitaires de France, pp. 206-226.

Mauger G., Poliak C. (2000), «Lectures : masculin/ féminin », Regards sociologiques, $\mathrm{n}^{\circ} 19$, pp. 115-140.

MazAde O. (2005), «Cellules de reclassement et individualisation du traitement du chômage. Le cas de Metaleurop Nord et des Houillères du Nord», Revue de l'IRES, $\mathrm{n}^{\circ} 47$, pp. 195-214.

Millet M., Thin D. (2012), «L'ambivalence des parents de classes populaires à l'égard des institutions de remédiation scolaire», Sociétés contemporaines, $n^{\circ} 86, \mathrm{pp}$. 59-83.

Pochic S. (2001a), Les cadres à l'épreuve de l'employabilité. Le chômage des cadres dans les années 1990 en France, thèse de Sociologie, Université Aix-Marseille II.

Pochic S. (2001b), «La menace du déclassement. Réflexions sur la genèse et l'évolution des projets professionnels», Revue de l'IRES, n 35, pp. 61-88.

Ricoeur P. (1990), Soi-même comme un autre, Paris, Seuil.

SAlah-Eddine L. (2010), «La reconnaissance du harcèlement moral au travail : une manifestation de la "psychologisation” du social?», SociologieS [En ligne], Premiers textes, mis en ligne le 27 décembre 2010, consulté le 28 mars 2013.

STEvens H. (2007), «Destins professionnels des femmes ingénieures. Des retournements inattendus », Sociologie du travail, vol. 49, $\mathrm{n}^{\circ} 4$, pp. 443-463.

Stevens H. (2008), «Quand le psychologique prend le pas sur le social pour comprendre et conduire des changements professionnels», Sociologies pratiques, $\mathrm{n}^{\circ} 17$, pp. 1-11.

Stevens H. (2010), «Les informaticiens de R\&D et les mutations du marché de l'informatique», in Boussard V., Demazière D., Milbum P. (dir), L'injonction au professionnalisme. Analyses d'une dynamique plurielle, Rennes, Presses universitaires de Rennes, pp. 41-50.

STRAuss A.L. (1992), La trame de la négociation : sociologie qualitative et interactionnisme, Paris, L'Harmattan.

VRancken D., Macquet C. (2006), Le travail sur soi. Vers une psychologisation de la société?, Paris, Belin, coll. «Perspectives sociologiques».

ZunE M. (2002), La constitution des trajectoires professionnelles. Le cas des professionnels des TIC face au modèle des "carrières nomades", Thèse doctorale de sociologie, Université de Liège. 